\title{
Câncer de mama no Brasil: medicina e saúde pública no século $X X$
}

\section{Breast cancer in Brazil: medicine and public health in 2oth century}

\author{
Luiz Antonio Teixeira ${ }^{a, b}$ \\ (D) https://orcid.org/0000-0001-8871-0928 \\ E-mail: luiz.teixeira®fiocruz.br \\ Luiz Alves Araújo Neto ${ }^{a}$ \\ (D) https://orcid.org/0000-0001-7965-2957 \\ E-mail: luizalvesanळhotmail.com \\ a Fundação Oswaldo Cruz. Casa de Oswaldo Cruz. Rio de Janeiro, \\ RJ, Brasil. \\ b Universidade Estácio de Sá. Programa de Pós-Graduação em \\ Saúde da Família. Rio de Janeiro, RJ, Brasil.
}

\section{Resumo}

Neste artigo, discutimos os caminhos que levaram o câncer de mama ao estatuto de grande problema de saúde pública e tópico constante de debates pela sociedade brasileira. Tendo como marco temporal o século XX, traçamos um amplo perfil das transformações nas abordagens ao câncer de mama no Brasil com base no desenvolvimento de novas tecnologias diagnósticas e terapêuticas e no jogo de forças entre diferentes grupos relacionados à sua implantação. Mostramos como, a partir do desenvolvimento de tecnologias médicas de diagnóstico precoce, deu-se mais atenção ao processo de prevenção à doença, implicando controvérsias sobre a melhor forma de implementar as práticas de prevenção. A análise se caracteriza como um estudo qualitativo de abordagem histórica, dialogando com trabalhos da história da medicina e dos estudos sociais das ciências. A investigação é pautada em fontes médicas (revistas especializadas, anais de congressos, teses, manuais); documentos institucionais e legislativos; jornais e revistas leigas; e um conjunto de entrevistas realizadas pelo projeto "História do Câncer: Atores, Cenários e Políticas Públicas" (Fundação Oswaldo Cruz/ Instituto Nacional de Câncer).

Palavras-chave: Câncer de Mama; Cirurgia; Rastreamento; Controle; Saúde Pública; Prevenção.

\section{Correspondência}


This study discusses the trajectory of breast cancer to becoming a major public health issue and constant topic of discussions in Brazilian society. We trace an extensive profile on the changes in approach concerning breast cancer in Brazil, considering the development of new diagnostic and therapeutic technologies and the power struggles between the different groups involved in their implementation. We show that with new technologies major attention was directed to the prevention of breast cancer, which led to controversies on how it would be done. The analysis is framed as a qualitative study with a historical approach based on studies of medical history and the Social Sciences. The data were collected from medical sources (specialized journals, congress reports, medical thesis and books); institutional and legislative documents; magazines and newspapers; and a corpus of interviews made by the project "History of Cancer: Actors, Scenarios and Public Policies" (Fiocruz/INCA).

Keywords: Breast Cancer; Surgery; Screening; Control; Public Health; Prevention.
No século XX ocorreram transformações radicais nas formas de compreender, representar e abordar o câncer. De agravo inespecífico, incurável e de baixa incidência, ele se transformou num mal cada vez mais conhecido, presente e temido pelas sociedades. Diversos processos sociais contribuíram para tais mudanças: o desenvolvimento do conhecimento médico, novas tecnologias diagnósticas e terapêuticas, transições demográficas e epidemiológicas, avanço da urbanização e industrialização, especialização médica etc. No Brasil, as primeiras iniciativas médicas de atuação contra a doença surgiram na década de 1920, mas somente nos anos 1940, no contexto de desenvolvimento de novas tecnologias diagnósticas e terapêuticas e de ampliação da medicina hospitalar, o câncer passou a ser visto como um problema de saúde pública, ou seja, uma doença que deveria ser alvo de ações coordenadas ou executadas por setores do Estado (Teixeira; Fonseca, 2007). Esse processo abrangeu diversos tipos de tumores, principalmente os de maior notabilidade (fosse pela mortalidade elevada ou pela carga simbólica a eles relacionada), mas repercutiu de maneiras distintas entre os vários cânceres. Discutiremos aqui os caminhos que levaram o câncer de mama ao estatuto de importante problema de saúde pública e tópico constante de debates pela sociedade brasileira.

Na primeira metade do século XX, discutia-se clinicamente sobre a detecção precoce da doença a partir das experiências dos médicos em serviço, analisando quais técnicas seriam mais apropriadas para o diagnóstico e discorrendo sobre o problema em periódicos de ginecologia ou em revistas dedicadas ao câncer de geral. Nesse período, pouco se sabia sobre sua incidência e mortalidade, e prevalecia o exame clínico como forma de diagnóstico. A partir dos anos 1940, com o desenvolvimento das primeiras instituições especializadas, começaram a surgir campanhas educativas postulando a importância do diagnóstico precoce como a única forma de possibilitar um tratamento mais efetivo. Duas décadas mais tarde, com o crescente otimismo com a possibilidade de resultados em rastreamento de câncer de mama equivalentes aos conseguidos com 
ações de rastreamento de câncer de colo, dava-se início a uma mudança no enquadramento da doença (Góes Júnior et al., 1977).

A partir dos anos 1970, a incorporação dos exames de imagem que permitiam a visualização de lesões mamárias bastante iniciais, a ampliação da mobilização feminina pela atenção à saúde da mulher e a reestruturação da saúde pública, fizeram com que a discussão sobre rastreamento de câncer mamário ganhasse força nas publicações especializadas, nos círculos médicos e no meio social. A princípio, os médicos achavam que o exame clínico era o procedimento mais apropriado para o rastreamento, por ser barato, não depender de tecnologia e aparelhos sofisticados e ser mais preciso que o autoexame. Na década de 1990, ainda tomando como base o modelo de rastreamento do câncer de colo e tendo como pano de fundo um novo sistema de saúde, agora de cunho universal, as propostas para o rastreamento a partir do uso massivo da mamografia começaram a ganhar força (Porto; Teixeira; Silva, 2013).

Hoje, o câncer de mama é o mais frequente na população feminina brasileira (com exceção do câncer de pele não melanoma). Segundo estimativas do Instituto Nacional de Câncer (Inca, 2019), em 2020, deverão ocorrer 66.280 casos novos de câncer de mama, configurando 29,7\% dos casos na população feminina. Apesar de décadas de iniciativas médicas e políticas públicas, as taxas de mortalidade da doença permanecem altas no Brasil. Estudos apontam que essas taxas se mantêm elevadas porque a doença ainda é diagnosticada em estádios avançados.

Neste artigo, traçamos um amplo perfil das transformações nas abordagens ao câncer de mama no Brasil com base no desenvolvimento de novas tecnologias de diagnóstico e terapêutica e no jogo de força entre diferentes grupos relacionados à sua implantação. A análise se caracteriza como um estudo qualitativo de abordagem histórica, que dialoga com trabalhos da história da medicina e dos estudos sociais das ciências acerca do processo de construção social de tecnologias médicas e suas relações com práticas de saúde (Rosenberg, 1992). A investigação é pautada em fontes médicas (revistas especializadas, anais de congressos, teses, manuais); documentos institucionais e legislativos; jornais e revistas leigas; e um conjunto de entrevistas realizadas pelo projeto "História do Câncer: Atores, Cenários e Políticas Públicas" (Fundação Oswaldo Cruz/Inca). ${ }^{1}$

\section{O mal das senhoras e a mastectomia radical}

As primeiras discussões sobre câncer de mama na medicina brasileira remetem ao início do século XX. Naquele momento, a doença era abordada de forma isolada por alguns médicos, principalmente através de relatos de casos clínicos em sessões de sociedades, como a Sociedade de Medicina e Cirurgia do Rio de Janeiro (Teixeira e Fonseca, 2007). Os tumores dos seios eram considerados comuns em mulheres idosas de classes mais abastadas; embora não houvesse explicação etiológica ou epidemiológica para esse fenômeno, eram vistos como incuráveis, havendo esperança de sobrevida somente através de um procedimento cirúrgico: a mastectomia radical.

A mastectomia radical recebeu o nome de William Halsted, cirurgião norte-americano que, na virada do século XIX para o XX, havia conseguido eliminar tumores cancerígenos de mulheres no John Hopkins Hospital, em Baltimore. Sua técnica consistia em cortes que extirpavam o tumor e uma considerável região em torno do seio, normalmente resultando na amputação de grande parte do tórax. A cirurgia halstediana, embora fosse considerada agressiva à época, foi transformada na principal ferramenta de tratamento, por fornecer melhores perspectivas do que qualquer outro procedimento tentado até então (Olson, 2005).

A introdução da mastectomia radical no Brasil foi o grande ponto de virada nas discussões sobre câncer de mama nas primeiras décadas do século XX, principalmente a partir de 1920. Artigos em periódicos especializados (Brazil Médico, Revista Brasileira de Medicina, Archivos Brasileiros de Medicina) e teses das faculdades do Rio de Janeiro (Conti, 1912) e Salvador discutiram os seus

\footnotetext{
1 Uma parte considerável das entrevistas discutidas na pesquisa sobre história do câncer de mama no Brasil foi recentemente publicada na coleção "Depoimentos para a História do Controle do Câncer no Brasil”.
} 
resultados, indicando a possibilidade de sobrevida das mulheres entre 3 e 5 anos, nos melhores cenários; um avanço considerável aos olhos dos cirurgiões no período (Santos Júnior, 1923). No entanto, para que as cirurgias tivessem sucesso, era necessário trazer as mulheres para o âmbito dos cuidados médicos, e fazê-lo tão logo a doença estivesse passível de ser percebida. Tal desafio não era de fácil resolução, pois, até o início do século XX, a medicina oficial compartilhava com outros sistemas de cura a preferência da população (Messora, 2017). No caso do câncer, o problema era agravado pela inexistência de uma terapêutica contra a doença vista como eficaz. Para os médicos, a reticência da população em procurar a ajuda médica resultava em diagnósticos de tumores em estágios bastante avançados (Santos Júnior, 1923).

Para os médicos do período, era necessário estar sempre alerta aos sinais do câncer de mama. Predominava a concepção de que que os tumores eram formados a partir de processos inflamatórios e lesões originárias de choques mecânicos, tornando fundamental a atenção a possíveis manchas, sangramentos, protuberâncias e outras "anomalias" nos seios. Esse formato de atenção constante (awareness) foi comum em outros países, como Estados Unidos e França, e esbarrava, entre outros aspectos, em tabus sobre o corpo feminino, dificultando a procura pelo profissional e o próprio conhecimento de si (Aronowitz, 2015).

A partir dos anos 1940, a criação do Serviço Nacional de Câncer, da Sociedade Brasileira de Cancerologia, bem como de instituições de assistência aos cancerosos pelo país, reconfigurou o campo de discussões sobre o câncer de mama. A ampliação da utilização da mastectomia, a introdução de novas tecnologias diagnósticas e a conformação de um campo cirúrgico mais direcionado à doença levou a novos debates sobre o combate aos tumores do seio.

\section{Câncer de mama e medicina hospitalar em meados do século $X X$}

O grande desenvolvimento da medicina hospitalar, determinado pela ampliação da medicina previdenciária, a partir dos anos 1940, possibilitou a disseminação das cirurgias para o câncer de mama. No que tange à saúde pública, as ações relacionadas à doença tiveram por base a criação de campanhas de esclarecimento quanto à necessidade de diagnóstico precoce. Nesse aspecto, o surgimento do Serviço Nacional de Câncer, em 1942, fortaleceu a noção de educação em saúde como melhor forma de controle da doença. No entanto, se a descoberta da doença em tempo hábil era vista como parte da responsabilidade das mulheres com o seu corpo, as ações estatais para o diagnóstico e tratamento ainda tinham um alcance muito curto, deixando sem cobertura a grande maioria das mulheres que precisavam de tratamento.

A ampliação dos conhecimentos e práticas dirigidas aos cânceres femininos, nesse período, teve como base a criação e o desenvolvimento de algumas instituições especializadas. O Instituto de Ginecologia da Faculdade de Medicina do Rio de Janeiro, O Serviço de Câncer Ginecológico da Cruz Vermelha e o Hospital Aristides Maltez (BA) foram as bases de organização do campo da cancerologia voltada para a saúde das mulheres (Lana, 2016). Dessas instituições surgiriam diferentes iniciativas de pesquisa, intervenção e organização do campo, direcionadas principalmente ao câncer de colo de útero que, em alguma medida, englobavam ações para a ampliação do diagnóstico do câncer de mama.

A organização do campo profissional ligado especificamente ao câncer de mama se inicia com a criação da Sociedade Brasileira de Patologia Mamária, em 1959. Fruto dos esforços do ginecologista e cancerologista do Inca, Alberto Coutinho, a sociedade veio à luz a partir da reunião de médicos em seu curso de especialização, realizado na Sociedade de Medicina e Cirurgia do Rio de Janeiro. Fundada na sede da SMCRJ, a Sociedade de Patologia Mamária teve como principal objetivo em seus primeiros anos a busca da transformação da mastologia em especialidade médica. A partir da década de 1970, então renomeada de Sociedade Brasileira de Mastologia (SBM), passou a contar com regionais em diversos estados do país (MG, RN, SC, etc.), oferecendo formação continuada a cirurgiões e ginecologistas, e passou a ter como objetivo o estímulo a ações públicas e privadas para o controle do câncer de mama no país. A SBM se tornou uma instituição de destaque na elaboração 
de políticas e normas direcionadas à doença (Porto; Teixeira; Silva, 2013).

Em relação às práticas direcionadas à doença, as maiores transformações começaram a partir da década de 1960. Nesse período, acompanhando as críticas à cirurgia radical de Halsted, surgidas nos países desenvolvidos, alguns cirurgiões brasileiros começaram a propor cirurgias mais conservadoras para câncer em estadiamentos iniciais. Mas independentemente do tipo de cirurgia proposto, o câncer de mama continuava a ser visto como uma doença fatal, cuja cirurgia somente ampliava a sobrevida da paciente (Ribeiro, 1965).

\section{Tecnologias médicas e a ampliação do diagnóstico}

A partir da década de 1960, dois fenômenos alteraram consideravelmente o enquadramento dos tumores do seio pela medicina e saúde pública brasileira. Por um lado, a introdução de novas técnicas de diagnóstico ampliou as discussões e expectativas em torno da realização do diagnóstico precoce. Por outro, a qualificação desse tipo de câncer como um problema típico de regiões urbanizadas mais desenvolvidas, existentes desde o início do século XIX, foi reforçada por estudos epidemiológicos, ampliando as preocupações com a doença em alguns estados e cidades do país (Araújo Neto, 2019).

Desde o começo do século XX, a medicina ocidental buscou métodos eficazes de detectar tumores nos seios, uma vez que a análise clínica possui limitações na capacidade de localizar nódulos pequenos. Além disso, o recrudescimento do senso de objetividade científica na medicina, privilegiando a visualização eletrônica e digital e leituras bioquímicas e citológicas do corpo, impulsionava a criação de tecnologias de diagnóstico por imagem, tendo como base o aparelho de radiografia. No Brasil, algumas tecnologias eram testadas desde os anos 1940, pari passu com a incorporação da radiologia à prática médica nacional. Procedimentos como a aeromamografia, consistindo na inserção de ar no seio para destacamento de bloqueios na glândula por imagem radiológica, eram testados pelos médicos em seus serviços privados; mas o alto custo dos aparelhos, as dificuldades em estabelecer uma semiologia específica para a doença e a própria falta de capacitação do olhar clínico para o diagnóstico por imagem impediram a disseminação dos novos procedimentos pelo país (Coutinho, 1941).

Nos anos 1960, a preocupação com o diagnóstico precoce foi acompanhada pela introdução de várias novas técnicas ao cotidiano de alguns serviços públicos e privados. Em capítulo de um volumoso manual de cancerologia publicado em 1967, o ginecologista paulista João Sampaio Góes Júnior (1967) indicou a existência de cinco métodos viáveis para o diagnóstico precoce de tumores na mama: o exame clínico; a biópsia; a citologia; a transiluminação; e a mastografia. A primeira alternativa era mais comum à prática médica do período, estando diretamente ligada à expertise dos clínicos; a biopsia e a citologia estavam na órbita dos patologistas. A transiluminação e a mastografia eram técnicas de visualização por imagem; a primeira resultante da aplicação de luz intensa em quarto escuro, destacando sombras em regiões com nódulos; e a segunda, por sua vez, era a radiografia da mama, precursora da mamografia.

Embora considerasse bastante relevante a existência de cada vez mais "armas para o combate" à doença, o médico paulista apostava na primazia do exame clínico das mamas como ferramenta correta para o trabalho:

O fator principal para o diagnóstico precoce do carcinoma mamário, reside na propedêutica, minuciosa e sistemática, das mamas de todas as mulheres e em todas as consultas. Devemos lembrar que grande número de tumores, benignos ou malignos, são descobertas de exame e ignorados por suas portadoras. Devem os médicos, em todas as oportunidades, procurar qualquer sinal que possa sugerir quadro patológico, principalmente do carcinoma mamário. Os obstetras que acompanham suas clientes, em frequentes consultas de pré-natal, devem examinar as mamas com o máximo cuidado, pois o ingurgitamento glandular da gestação pode mascarar a presença de pequenos nódulos e, só tardiamente, autorizar o diagnóstico. (Góes Júnior, 1967, p. 469) 
O argumento de Góes Júnior estava relacionado à ideia de uma rotinização dos exames clínicos, com a frequência regular das mulheres nos gabinetes médicos. Esse perfil era limitado às classes médias urbanas, que tinham acesso ao sistema previdenciário através dos institutos de assistência por classe trabalhista. 0 médico dialogava com a interpretação de que determinados tipos de câncer, como o de mama, seriam específicos de regiões urbanas e industrializadas, nas quais as mulheres teriam acesso a melhores condições sanitárias e de higiene, permitindo o envelhecimento; além de terem um estilo de vida associado ao risco de câncer (Mirra; Cole; MacMahon, 1971).

Para outros tipos de câncer, como do colo do útero, o enquadramento era inverso: um problema da pobreza, de más condições de saúde e dificuldade de acesso aos serviços. Neste caso, a percepção de que um grande número de casos estava fora da esfera da medicina previdenciária foi ponto importante na adoção de uma nova estratégia para redução da mortalidade, baseada na utilização de exames diagnósticos em população sadia de maior risco, com o intuito de detectar as lesões precursoras da doença no estágio mais inicial possível. Essa estratégia, (rastreamento ou screening), teve como técnica central o exame citopatológico (Papanicolaou), e foi posta em prática inicialmente em programas de saúde pública ligados a universidades (Teixeira, 2015).

No Brasil, de forma semelhante ao que vinha acontecendo na Europa e nos Estados Unidos, a noção de que o rastreamento de câncer de colo poderia ser o modelo para outras doenças fez com que logo se pensasse em utilização da mesma metodologia de prevenção específica para o câncer de mama (Löwy, 2010). Independente do fato de uma campanha de rastreamento de câncer de mama poder se dar a partir do autoexame, do exame clínico ou, posteriormente da mamografia, a possibilidade de rastrear mulheres para evitar a doença abria uma nova perspectiva para a saúde pública brasileira.

\section{A introdução da mamografia e as primeiras propostas de rastreamento}

Os anos 1970 marcariam uma grande transformação na busca de controle do câncer de mama. No contexto internacional, desde a década anterior, a ampliação da utilização de diversas tecnologias para a busca de tumores muito pequenos, e a cada vez maior aceitação das imagens radiográficas para o diagnóstico precoce da doença, já se faziam presentes. Mas a partir de então, ensaios clínicos mostrando o valor do rastreamento mamográfico na diminuição das taxas de mortalidade pela doença, transformam essa tecnologia em padrão ouro no controle do câncer de mama. No Brasil, nesse mesmo período, os exames de imagem passaram à órbita de interesse dos médicos da Sociedade de Patologia Mamária e dos ginecologistas. A entrada no país dos primeiros mamógrafos, ainda em 1970, despertou a curiosidade na nova tecnologia e criou novas demandas para a saúde pública.

O primeiro mamógrafo foi importado por Sampaio Góes para o Instituto Brasileiro de Estudos e Pesquisas em Oncologia e Obstetrícia (Ibepog), parceria público/privada por ele dirigida. Comprado da Companie Generale de Radiologie (CGR), o mamógrafo foi inicialmente instalado no consultório privado de Sampaio Góes, para lhe permitir a ampliação dos conhecimentos sobre o uso do aparelho (Teixeira, 2015). Posteriormente, Sampaio Góes enviaria seu filho, João Carlos Guedes Sampaio Góes, para a França para se especializar na nova tecnologia.

Em 1972, o Centro de Pesquisas Luiza Gomes de Lemos também adquiriu um mamógrafo e começou a realizar exames de rastreamento de câncer de mama, de forma oportunística nas mulheres que eram consultadas em seu centro ginecológico. Em pouco tempo, o centro também passou a elaborar campanhas de rastreamento da doença em diversos bairros da Guanabara a partir de um caminhão transformado em unidade volante. Nos bairros visitados, as comunidades eram instruídas, por meio de palestras, sobre a necessidade do autoexame periódico e do exame mamográfico. Em pouco tempo, o número de atendimentos no centro foi ampliado fortemente em virtude dos programas feitos nos diferentes bairros. Em 1974, o centro reformou as instalações de radiografia, adquiriu um novo mamógrafo e um aparelho de termografia. Segundo o relatório daquele ano, em 24 meses, em torno de 12 mil mulheres passaram por exames mamográfico em suas dependências (Temperini, 2012). 
No âmbito das possibilidades de prevenção, a mamografia de rastreamento era vista por muitos médicos como "a tecnologia do futuro" (Mesa..., 1976). Apesar desse otimismo, e mesmo do fato de já existirem iniciativas de rastreamento mamográfico no país (como o observado no parágrafo precedente), a visão do controle do câncer tinha no rastreamento por autoexame e exame clínico o maior número de defensores. Diversos eventos deixam claras essas escolhas.

Ainda em 1973, Sampaio Góes elaborou um programa piloto de rastreamento do câncer de colo e de mama em São Caetano, numa parceria entre o Instituto Brasileiro De Controle Do Câncer, a Secretaria de Saúde do Estado de São Paulo e a Secretaria de Higiene de São Caetano. O protocolo desenvolvido previa exame físico realizado por não médicos; exame clínico nos casos triados; mamografia; punção; e citologia (Góes Júnior et al., 1977). Em 1974, a Divisão Nacional do Câncer criou o Grupo Operativo de Controle do Câncer Mamário. O grupo recomendava o exame clínico das mamas em período semestral, ou de quatro em quatro meses nas pacientes de alto risco (Rostein, 1976). Três anos depois, no IV Congresso da Sociedade Brasileira de Mastologia, ocorrido em Campinas, vários estudos se detiveram na apreciação da mamografia como forma de exame de rastreamento, sendo que a maioria deles afirmava que, embora a técnica fosse eficiente, seu preço e necessidade de material humano para operá-la fazia com que não fosse a melhor forma para a detecção da doença, sendo o autoexame e o exame clínico os caminhos a serem seguidos (Barbosa, 1977, p. 85).

É importante frisar que, embora os médicos nacionais defendessem o uso do autoexame e do exame clínico como principal forma de controle da doença, o desenvolvimento da mastologia e a formação de especialistas seguiam caminho contrário. 0 melhor exemplo disso é o fato de que o convidado de honra do congresso foi o médico Philip Strax, que havia desenvolvido vários estudos comprovando a importância da mamografia na redução da mortalidade por câncer de mama, e criado o primeiro serviço móvel de mamografia na cidade de Nova York.

\section{Medicina de risco e o gênero do câncer de mama}

Nos anos 1970, os primeiros dados estatísticos obtidos pelos registros de câncer de base hospitalar e populacional possibilitaram o aprofundamento da discussão acerca dos fatores de risco associados ao câncer de mama e de estratégias de prevenção primária da doença (Araújo Neto, 2019). O fortalecimento da visão do câncer de mama como um problema de saúde do mundo urbano, atingindo principalmente mulheres de classe média acima dos 50 anos, serviu de parâmetro para debates sobre a doença e, sobretudo, as doentes.

Conforme aponta Aronowitz (2015), o desenvolvimento da medicina de risco (riskymedicine), orientada pelo conhecimento epidemiológico, teve como grandes consequências o mapeamento de estilos de vida e substâncias capazes de aumentar as chances de adoecimento e a convergência da experiência do risco com a da doença, principalmente para enfermidades crônico-degenerativas.

No que diz respeito ao estilo de vida da mulher urbana, dois aspectos que constituíam uma imagem do feminino ganharam destaque nesse período: a maternidade e o uso de pílulas anticoncepcionais. Por um lado, a gravidez e o aleitamento materno foram qualificados como processos que ajudam as mulheres na prevenção do câncer de mama; entretanto, a mulher não deveria ter muitos filhos (multípara), pois representaria fator de risco para câncer do colo do útero (Amamentar..., 1971, p. 3). Por outro lado, o uso das pílulas contraceptivas foi motivo de grande debate nos anos 1970, principalmente a partir de proposta do Congresso Nacional de proibir a venda do fármaco no país.

Os posicionamentos sobre o assunto eram heterogêneos. De modo geral, considerava-se que o uso excessivo das pílulas aumentaria as chances de as mulheres desenvolverem câncer de mama, com o risco variando de acordo com o tempo de consumo do produto. Com base nisso, alguns médicos, como o ginecologista Salles Soares, professor da Faculdade de Medicina da Guanabara, sugeriam "que as pílulas, bem como o cigarro, fossem postas na lista de produtos carcinogênicos pelo INCA" (Médico..., 1970, p. 7). 
A discussão sobre os riscos representados pelas pílulas anticoncepcionais prosseguiu durante os anos 1970, tendo como chave principal a proibição do uso. Apesar disso, não houve medidas proibitivas por parte do Ministério da Saúde no período, somente a medicalização da pílula a partir da obrigatoriedade de apresentar a receita para sua obtenção. No contexto de fortalecimento da visão da maternidade como uma escolha da mulher e da necessidade de controle da natalidade de países em desenvolvimento, os discursos sobre a ampliação do risco de câncer de mama em virtude da utilização das pílulas tiveram eco limitado.

No que tange à convergência da experiência entre risco e doença no câncer de mama, as consequências da medicina de risco recaem diretamente nas relações das mulheres com seu próprio corpo, que cada vez mais é visto como doente na medida que se enquadra no maior risco de adoecimento. Nos últimos anos, ganhou destaque a prática da mastectomia profilática, ou seja, retirada dos seios para prevenir a formação de tumores. Entretanto, práticas desse tipo não são novas, e ganharam espaço nos debates médicos entre os anos 1960 e 1980. Nesse período, um dos fatores de riscos sugeridos pelos estudos epidemiológicos dizia respeito à carga hormonal das mulheres, levando a sugestão de intervenções químicas ou mesmo cirúrgicas, a exemplo da ooforectomia (retirada do ovário), como forma de reduzir as chances de adoecimento.

\section{Câncer de mama e saúde pública}

Nos anos 1980, num contexto de enfraquecimento do regime de exceção vivido no Brasil, o movimento feminista, que havia surgido no país de forma tímida na década anterior, ganhou mais força, se institucionalizou e passou a buscar participação na formulação das políticas públicas. Para além das demandas por igualdade de oportunidades, sua nova agenda continha questões relacionadas à violência contra a mulher e pela saúde (Pinto, 2003). 0 movimento feminista alterou a forma de se pensar a relação do Estado com a saúde das mulheres. Até então, inexistia uma política específica dirigida à saúde deste grupo, sendo que o Estado resumia suas preocupações ao papel das mulheres na reprodução, incentivando, de forma velada, ações intervencionistas, à época preconizada pelos Estado Unidos. Foi justamente no âmbito das discussões sobre corpo e reprodução que as questões sobre a saúde da mulher afloraram no discurso feminista (Mesquita, 2010).

Em 1983, quando o governo dos generais anunciou ao congresso a necessidade de aprofundar as discussões sobre o crescimento populacional, militantes feministas, grupos voltados para a reforma do sistema de saúde e parte da classe médica crítica à visão puramente biologicista da saúde, se aliaram na busca de constituir um programa mais amplo, que não resumisse a saúde da mulher a questões reprodutivas. Em 1984, como resultante desse processo, surgiu o Programa de Assistência Integral à Saúde da Mulher (PAISM). 0 programa propunha ações educativas, preventivas, de diagnóstico, tratamento e recuperação. Previa assistência à mulher em clínica ginecológica, no pré-natal, parto e puerpério, no climatério, em planejamento familiar, com doenças sexualmente transmissíveis, câncer de colo de útero e de mama, além de outras necessidades identificadas a partir do perfil populacional das mulheres.

Os ideais que conformaram a política de atenção integral à saúde da mulher foram gestados no mesmo núcleo institucional que pôs à frente as primeiras campanhas de rastreamento de câncer de colo de útero em Campinas. Os técnicos da Universidade de Campinas (Unicamp) foram os principais formuladores do que viria a ser o PAISM. A partir de uma interlocução com o movimento de mulheres, eles buscaram integrar ações pela saúde de cunho médico, como o controle do câncer de colo de útero e das mamas a outras mais ampliadas, direcionadas ao bem-estar e a aspectos psicológicos e emocionais das mulheres (Rosa, 2011).

O grupo de pesquisadores de Campinas, que deu início à demanda por programas de saúde pública direcionados ao câncer de mama ,era liderado pelo médico Jose Aristodemo Pinotti, tendo como um de seus principais colaboradores Luiz Carlos Zeferino, outros médicos do centro de Saúde da Mulher, da Universidade de São Paulo e do Centro de Atenção Integral à Saúde da Mulher (CAISM), da Unicamp. Tendo como referência as propostas 
relacionadas ao planejamento e à organização de serviços hierarquizados, baseados na organização e distribuição dos cuidados nos diferentes níveis hierárquicos da saúde, os pesquisadores das duas universidades propunham a ampliação das políticas públicas em relação às mulheres a partir de tecnologias leves e práticas de maior alcance, muitas delas relacionadas à atenção primária (Zeferino; Pinotti; Teixeira, 1987). Nesse sentido, postulavam que os programas de rastreamento de câncer de mama por mamografia não se adequavam à realidade econômica do nosso país, e a estratégia mais adequada para o controle da doença era o rastreamento a partir de exames clínicos e autoexames (Pinotti et al., 1992).

Outro grupo a defender ideias semelhantes em relação ao controle do câncer de mama teve como base o Inca. Em 1988, com a criação do Sistema Único de Saúde (SUS), o Inca tornou-se responsável pelas políticas de câncer no país, passando a coordenar o Programa de Oncologia (Pro-Onco), criado pelo governo federal em 1987 com o objetivo de ampliar as ações de prevenção e diagnóstico precoce de câncer em nível nacional. A partir desse período, os artigos de pesquisadores do Pro-Onco passaram a reafirmar a importância do rastreamento mamográfico na diminuição da mortalidade por câncer de mama. Seguindo as postulações da Union for International Cancer Control (UICC) e da Organização Panamericana de Saúde (OPS), também propunham que em países em desenvolvimento o rastreamento deveria ser precedido de estudos e estruturas que mostrassem sua viabilidade. Nesse sentido, indicavam os exames clínicos e autoexames como forma de detecção precoce do câncer de mama (Lopes; Abreu; Gadelha, 1993).

\section{Reconfigurando velhas questões: câncer de mama e a estruturação do SUS}

No final da década de 1980, uma reforma sanitária deu origem a um novo sistema de saúde no Brasil. De caráter público e universal - embora convivendo com um subsistema privado, responsável por atender $25 \%$ da população - o SUS pautou um novo arranjo na dinâmica de definição de estratégias na área, com maior participação da sociedade, abrindo a possibilidade de lobbies e articulações para a mobilização de agendas de grupos específicos.

No caso do câncer de mama, o cenário moldado pelo novo sistema de saúde e pelo desenvolvimento da medicina baseada em evidência e dos protocolos médicos permitiu a reconfiguração de questões já colocadas há muito tempo, entre as quais se destacam o uso de práticas terapêuticas conservadoras e as proposições sobre o rastreamento para câncer de mama no país.

A combinação da mastectomia a outras técnicas terapêuticas, como a quimioterapia, a hormonioterapia e a radioterapia, permitiu um aprofundamento da crítica ao método halstediano e a aplicação de procedimentos menos agressivos. A preocupação com a qualidade de vida das pacientes ganhou mais atenção das instituições de assistência, com a atuação de profissionais da área psicológica, do serviço social e da enfermagem no cuidado às pacientes mastectomizadas (Bervian; GirardonPerlinni, 2006).

O rastreamento, por sua vez, configurou um cenário de fortes debates sem a definição de consenso sobre qual estratégia adotar para o controle do câncer de mama e como estabelecer uma política para tal. Para uns, era necessária a criação de um programa de rastreamento organizado em nível nacional. Outros propunham a manutenção de atividades de rastreamento oportunístico. No que tange à indicação do exame também havia dissenso: para os médicos da saúde pública, a faixa etária dos 50 aos 69 anos era a população a quem o exame deveria ser direcionado. Alguns especialistas, no entanto, propunham que as mulheres deveriam fazer exames anuais a partir dos 40 anos.

Nesse contexto de incertezas, em 2000, o Inca vinculou o câncer de mama ao programa de rastreamento nacional de câncer de colo que vinha coordenando - o Viva Mulher. De início, a proposta era diagnosticar os tumores clinicamente detectáveis por meio do autoexame e do exame clínico das mamas. A partir de 2002, essa diretriz mudou para o uso da mamografia, com o objetivo de detectar os tumores não detectáveis clinicamente. Embora o Ministério da Saúde tenha adquirido 50 mamógrafos 
e distribuído para algumas secretarias municipais de saúde com essa finalidade, a iniciativa não gerou um programa de rastreamento consolidado (Porto; Teixeira, 2013).

Em 2003, o Ministério da Saúde, o INCA e a Sociedade Brasileira de Mastologia realizam uma ampla oficina de trabalho, visando alinhar as recomendações referentes ao controle do câncer de mama. Dela participaram técnicos do Ministério, gestores, pesquisadores e representantes de sociedades científicas e de entidades de mulheres. $\mathrm{O}$ documento de consenso resultante recomendou o rastreamento por meio do exame clínico das mamas anual para mulheres a partir de 40 anos e o exame mamográfico bienal para mulheres de 50 a 69 anos. Para mulheres de risco elevado, a recomendação é a realização do exame clínico das mamas e mamografia anuais, a partir dos 35 anos de idade. Além disso, enfatizou a necessidade de garantir acesso ao diagnóstico, tratamento e seguimento para todas as mulheres com alterações nos exames realizados. Na esteira da publicação do documento de consenso, o Ministério da Saúde editou novas diretrizes que reforçavam a importância do controle do câncer de mama (INCA, 2004).

Apesar de sua denominação, o documento de consenso não fechou as controvérsias sobre a prevenção do câncer de mama no país. A recomendação de rastreamento anual para mulheres entre 50 e 69 anos não foi aceita por diversas sociedades médicas, que permaneceram postulando que o rastreamento deveria se dar a partir dos 40 anos. Nesse momento, a maior mobilização de grupos alinhados às propostas dos produtores privados de insumos para a saúde, relativas à ampliação do uso da mamografia, reconfiguram a arena de discussões sobre o controle do câncer de mama e, especificamente, o uso da mamografia. Se, até então, os debates sobre rastreamento e as possibilidades de utilização da mamografia eram circunscritos a uma parcela do campo médico, em especial ao Inca, no século XXI o tema ganhou maior amplitude, principalmente devido ao surgimento das primeiras associações de pacientes de câncer de mama (Porto; Teixeira, 2013). A partir de 2006, com a criação da Federação Brasileira de Instituições Filantrópicas de Apoio à Saúde da Mama (Femama), as demandas das diferentes associações de pacientes e das associações de especialistas em relação à ampliação da faixa etária do exame de rastreamento e de maior cobertura do exame se intensificaram, dando um novo perfil ao problema do câncer de mama.

\section{Considerações finais}

Neste artigo, discutimos as transformações nas abordagens da medicina e da saúde pública sobre o câncer de mama no Brasil ao longo do século XX. A introdução de novas tecnologias e saberes do campo médico à atenção oncológica teve papel fundamental na definição de formas de pensar a doença e estabeleceu pontos de debate que repercutem no cenário atual do controle do câncer de mama no país.

Mais especificamente, exploramos como as mudanças nas abordagens sobre a doença estiveram condicionadas às negociações de diferentes atores e grupos sociais em torno das tecnologias. Na primeira metade do século XX, a autoridade dos cirurgiões quanto à prática da mastectomia delimitou não somente um espaço de atuação, mas também um universo de relações entre os médicos especialistas, a saúde pública e as mulheres doentes, dominadas pelos cirurgiões. Principalmente a partir dos anos 1970, porém, a entrada em jogo de um novo conjunto de conhecimentos - vinculado à medicina de risco, à epidemiologia e à biomedicina - e de tecnologias, principalmente as de visualização da mama, reorientou o eixo de discussões sobre a doença para a prevenção. Articulados às demandas dos movimentos de mulheres, esses saberes e técnicas possibilitaram a formulação de discussões específica sobre câncer e saúde feminina.

Entretanto, como também foi explorado no artigo, a entrada de novos atores implicou a ampliação do escopo de disputas em torno das tecnologias, fortalecendo controvérsias sobre como a prevenção do câncer de mama deve ser realizada no Brasil. Nessa discussão, as diferentes agendas do setor público, da medicina privada e das mulheres enfermas colocam impasses que não puderam ser resolvidos por meio de consensos, recomendações e protocolos.

Nas duas primeiras décadas do século XXI, o debate sobre o câncer de mama tem ganhado mais espaço na cena pública, principalmente devido 
ao Outubro Rosa, evento de intensificação de campanhas educativas e outras ações relacionadas à doença. Em diversos momentos, o problema do câncer de mama aparece caracterizado de maneira simplificada, como fruto de interesses do setor privado, da ineficiência do poder público, ou mesmo de uma ignorância da população quanto aos procedimentos para prevenção, diagnóstico e tratamento dos tumores. Acreditamos que uma leitura histórica e sociológica das abordagens sobre esse tipo de câncer, tão importante no cotidiano da população feminina brasileira, apresente caminhos de discussão diferentes, levando em conta a diversidade de visões, projetos e preocupações envolvidas no controle do câncer de mama.

Na última década, a questão do rastreamento para câncer de mama teve maior força social em relação a outros aspectos de controle da doença, sendo o principal tema debatido no campo da saúde pública e o mais veiculado na imprensa. No entanto, outras questões, como o acesso ao tratamento e a novas tecnologias de ponta (exemplo das terapias alvo), além das mobilizações em torno dos cuidados com a mulher mastectomizada fora do tradicional âmbito da medicina e da saúde, têm ganhado espaço através de associações de pacientes, redes sociais, e outros fóruns. 0 dinamismo das discussões e as preocupações com o câncer de mama demonstram a importância que a doença assumiu na sociedade, e demandam mais pesquisas acerca das faces sociais e históricas do câncer no país.

\section{Referências}

AMAMENTAR filho ajuda a evitar o câncer no seio. Correio da Manhã, Rio de Janeiro, p. 3, 3 set. 1971.

ARAÚJO NETO, L. A. Prevenção do câncer no Brasil: mudança conceitual e continuidade institucional no século XX. 2019. Tese (Doutorado em História das Ciências e da Saúde) - Casa de Oswaldo Cruz, Fiocruz, Rio de Janeiro, 2019.

ARONOWITZ, R. Risky Medicine: our quest to cure fear and uncertainty. Chicago: University of Chicago Press, 2015.

BARBOSA, L. Valor do exame clínico e da autopalpação. In: CONGRESSO BRASILEIRO DE
MASTOLOGIA, 4., 14-18 fev. 1977, Campinas. Anais... Campinas: Unicamp, 1977. p. 77-85.

BERVIAN, P.; GIRARDON-PERLINI, N. A

família (com)vivendo com a mulher/mãe após a mastectomia. Revista Brasileira de Cancerologia, Rio de Janeiro, v. 52, n. 2, p. 121-128, 2006.

CONTI, S. Câncer do seio. 1912. Tese - Faculdade de Medicina do Rio de Janeiro, Rio de Janeiro, 1912.

COUTINHO, A. Cancer da Mama. Annaes de Ginecologia, Rio de Janeiro, ano VI, v. 11, p. 205-241, 1941.

GÓES JÚNIOR, J. S. Diagnóstico do câncer de mama. In: ROXO NOBRE, M.; JUNQUEIRA, A. Cancerologia prática. São Paulo: Pronciex, 1967. v. 2, p. 6o-92.

GÓES JÚNIOR, J. S. et al. Programa de detecção em massa do câncer da mama - I.B.C.C 1973/1976. In: CONGRESSO BRASILEIRO DE MASTOLOGIA, 4., 14-18 fev. 1977, Campinas. Anais... Campinas: Unicamp, 1977. p. 429-441.

INCA - INSTITUTO NACIONAL DE CÂNCER. Controle do câncer de mama: documento do consenso. Rio de Janeiro, 2004.

INCA - INSTITUTO NACIONAL DE CÂNCER. $O$ controle do câncer de mama no Brasil: trajetórias e controvérsias. Rio de Janeiro, 2018. (Série Depoimentos para a História do Controle do Câncer no Brasil).

INCA - INSTITUTO NACIONAL DE CÂNCER. Estimativa 2020: incidência de câncer no Brasil. Rio de Janeiro, 2019.

LANA, V. Organização da especialidade médica e controle do câncer do colo do útero no Brasil: o Instituto de Ginecologia do Rio de Janeiro em meados do século XX. História, Ciências, Saúde, Manguinhos, v. 23, n. 3, p. 683-701, 2016.

LOPES, E.; ABREU, E.; GADELHA M. Detecção do câncer de mama: atualização e recomendações. Revista Brasileira de Cancerologia, Rio de Janeiro, v. 39, n. 1, p. 21-26, 1993.

LÖWY, I. Preventive strikes: women, precancer, and prophylactic surgery. Baltimore: Johns Hopkins University Press, 2010 
MÉDICO afirma que pílula é grande perigo como agente cancerígeno. Correio da Manhã, Rio de Janeiro, p. 7, 25 jan. 1970.

MESA redonda: Avaliação dos métodos propedêuticos na prevenção e diagnóstico precoce do câncer de mama. Arquivos de Oncologia, [S.l.], v. 17, n. 1, 1976.

MESQUITA, C. C. Saúde da mulhere redemocratização: idéias e atores políticos na história do PAISM. 2010. Dissertação (Mestrado em História das Ciências e da Saúde) - Casa de Oswaldo Cruz, Fundação Oswaldo Cruz, Rio de Janeiro, 2010.

MESSORA, E. A construção de um novo mal: representações do câncer em São Paulo, 1892-1953 2017. Dissertação (Mestrado em Medicina Preventiva) - Faculdade de Medicina, Universidade de São Paulo, São Paulo, 2017.

MIRRA, A. P.; COLE, P.; MACMAHON, B. Breast cancer in an area of high parity: São Paulo, Brazil. Cancer Research, Chicago, v. 31, n. 2, p. 77-83, 1971.

OLSON, J. Bathsheba's breast: women, cancer, and history. Baltimore: Johns Hopkins University Press; 2005.

PINOTTI, J. A. et. al. Programa de controle do câncer da mama em países em desenvolvimento. In: PINOTTI, J. A. et al. (Org.). Avanços em oncologia. Volume II: Câncer de Mama. São Paulo: Ícone, 1992. p. 13-21.

PINTO, C. R. J. Uma história do feminismo no Brasil. São Paulo: Ed. Fundação Perseu Abramo, 2003 PORTO, M.; TEIXEIRA, L.; SILVA, R. Aspetos históricos do controle de câncer de mama no
Brasil. Revista Brasileira de Cancerologia, Rio de Janeiro, v. 59, n. 3, p. 331-339, 2013.

RIBEIRO, B. Tratamento conservador do câncer de mama. Arquivos de Oncologia, [S.l.], v. 5, n. 1, p. 144-146, 1965 .

ROSA, C. A. CAISM: a história de sua implantação. Campinas: Unicamp, 2011

ROSENBERG, C. Framing disease: studies in cultural history. New Brunswick: Rutgers University Press, 1992.

ROSTEIN, S. Diagnóstico precoce do câncer mamário. Ginecologia Brasileira, [S.l.], v. III, n. 1, p. 63-68, 1976.

SANTOS JÚNIOR, D. Ligeiras considerações sobre o câncer de mama e o methodo de Halsted. Tese apresentada à Faculdade de Medicina da Bahia. Salvador: Imprensa Oficial do Estado, 1923.

TEIXEIRA, L.; FONSECA, C. De doença desconhecida a problema de saúde pública: o INCA e o controle do câncer no Brasil. Rio de Janeiro: Ministério da Saúde, 2007.

TEIXEIRA, L. A. From gynaecology offices to screening campaigns: a brief history of cervical cancer prevention in Brazil. História, Ciência, Saúde, Manguinhos, v. 22, n. 1, p. 221-239, 2015.

TEMPERINI, R. Fundação das Pioneiras Sociais contribuição inovadora para o Controle do Câncer do Colo do Útero no Brasil (1956-1970). Revista Brasileira de Cancerologia, Rio de Janeiro, v. 58, n. 3, p. 339-349, 2012.

ZEFERINO, L.; PINOTTI, J; TEIXEIRA, L. O problema do câncer ginecológico e mamário. Anais Brasileiros de Ginecologia, Rio de Janeiro, v. 15, n. 3, p. 198-212, 1987.

\section{Contribuição dos autores}

Luiz Antonio Teixeira e Luiz Alves Araújo Neto contribuíram igualmente para a concepção, pesquisa de fontes e redação do manuscrito. Ambos revisaram o manuscrito e aprovaram a versão final.

Recebido: 08/09/2019

Aprovado: 09/06/2020 